\title{
Metal-induced stress in survivor plants following the end-Permian collapse of
}

\section{land ecosystems}

Daoliang $\mathrm{Chu}^{1 *}$, Jacopo Dal Corso ${ }^{1}$, Wenchao Shu ${ }^{1}$, Haijun Song ${ }^{1}$, Paul B. Wignall ${ }^{2}$, Stephen E.

Grasby $^{3}$, Bas van de Schootbrugge ${ }^{4}$, Keqing Zong ${ }^{6}$, Yuyang $\mathrm{Wu}^{1}$, and Jinnan Tong ${ }^{1}$

${ }^{1}$ State Key Laboratory of Biogeology and Environmental Geology, School of Earth Sciences, China University of Geosciences, Wuhan 430074, China

${ }^{2}$ School of Earth and Environment, University of Leeds, Leeds LS2 9JT, UK

${ }^{3}$ Geological Survey of Canada, Natural Resources Canada, Calgary, Alberta T2L2A7, Canada

${ }^{4}$ Department of Earth Sciences, Utrecht University, Utrecht 3584 CB, Netherlands

${ }^{5}$ Department of Earth Sciences, University of Oxford, South Parks Road, Oxford OXI 3AN, UK

${ }^{6}$ School of Earth Sciences, State Key Laboratory of Geological Processes and Mineral Resources, China University of Geosciences, Wuhan 430074, China

*Corresponding author: chudl@cug.edu.cn

\section{Borehole ZK4703}

The borehole ZK4703 $\left(25.54151^{\circ} \mathrm{N}, 104.28994^{\circ} \mathrm{E}\right)$ was drilled $15 \mathrm{~km}$ south of Fuyuan County, Xuanwei City, Yunnan Province in summer of 2014 by Coal Geology Bureau. It was one of dozens of boreholes drilled to explore the Permian coal reserves. It consists of Carboniferous, Permian and Early Triassic strata. The core of Xuanwei Formation (Late Permian), Kayitou Formation (Permian-Triassic transition) and lower part of Dongchuan Formation (Early Triassic) are currently stored at State Key Laboratory of Biogeology and Environmental Geology, China University of Geosciences (Wuhan).

\section{Palynological analysis}

36 samples have been collected from core ZK4703 of South China, across the Permian-Triassic transition. And all of them have been processed with the standard palaeopalynological method without the oxidation process. The residues were then mounted on permanent slides (ten slides for each sample) by using the medical neutral gum. The quantitative palynological analysis on 19 productive samples were counted as around 100 sporomorphs per sample (Table DR2). Those processes were carried on a Zeiss Axio Scope A1 microscope. All residues in sealed tubes with the antifungal process and permanent slides were stored at State Key Laboratory of Biogeology and Environmental Geology, China University of Geosciences (Wuhan).

\section{Trace element analysis}

$\mathrm{Al}$ and $\mathrm{Cu}$ contents of whole rock were conducted on Agilent 7700x ICP-MS at the Wuhan Sample-Solution Analytical Technology Co., Ltd.. $\mathrm{NH}_{4} \mathrm{HF}_{2}$-open-vessel acid digestion technique was 
used in this study in order to complete dissolute refractory minerals. The detailed sample-digesting procedure was as follows: (1) Sample powder (200 mesh) are placed in an oven at $105{ }^{\circ} \mathrm{C}$ for drying of 12 hours; (2) $50 \mathrm{mg}$ sample powder is accurately weighted and mixed with $200 \mathrm{mg}$ pure $\mathrm{NH}_{4} \mathrm{HF}_{2}$ powder in a 7-mL screw-top PFA vial; (3) The vials are then capped and heated at $230{ }^{\circ} \mathrm{C}$ in an electric oven for 3 hours; (4) After cooling, $2 \mathrm{~mL} \mathrm{HNO}_{3}$ are added, and the vials are capped and placed on a hot plate at $160{ }^{\circ} \mathrm{C}$. After 1 hour, the vials are opened and evaporated to near dryness at $160^{\circ} \mathrm{C}$ on the hot plate; (5) The final residue is then taken up in $1 \mathrm{~mL} \mathrm{HNO}_{3}, 1 \mathrm{~mL} \mathrm{MQ}$ water, and 1 $\mathrm{mL}$ internal standard solution of $1 \mathrm{ppm}$ In. The vials are recapped and heated for 6 hours at $120^{\circ} \mathrm{C}$ to obtain a clear solution; (6) The final solution was transferred to a polyethylene bottle and diluted to $100 \mathrm{~g}$ by the addition of $2 \% \mathrm{HNO} 3$ before ICP-MS analysis. The accuracy of $\mathrm{Al}$ and $\mathrm{Cu}$ of international standards BCR-2, BHVO-2 and AGV-2 are better than 5\% . 
Table DR1. $\mathrm{Hg}, \mathrm{Cu}$, TOC and $\mathrm{Al}$ concentrations, and $\mathrm{Hg} / \mathrm{TOC}, \mathrm{Hg} / \mathrm{Al}$ and $\mathrm{Cu} / \mathrm{Al}$ ratios from $\mathrm{ZK} 4703$.

\begin{tabular}{|c|c|c|c|c|c|c|c|c|c|c|c|c|c|}
\hline Sample & Height (m) & $\mathrm{Al}(\mathrm{ppm})$ & $\mathrm{Cu}(\mathrm{ppm})$ & TOC (\%) & $\mathrm{Hg}(\mathrm{ppb})$ & $\begin{array}{l}\mathrm{Hg} / \mathrm{TOC} \\
(\mathrm{ppb} / \%)\end{array}$ & $\mathrm{Hg} / \mathrm{Al}^{*} 10^{8}$ & $\mathrm{Cu} / \mathrm{Al}^{*} 10^{4}$ & $\begin{array}{l}\mathrm{Cu} / \mathrm{TOC} \\
(\mathrm{ppm} / \%)\end{array}$ & $\mathrm{Ni}(\mathrm{ppm})$ & $\mathrm{Pb}(\mathrm{ppm})$ & $\mathrm{Cr}(\mathrm{ppm})$ & $\mathrm{Zn}(\mathrm{ppm})$ \\
\hline$\overline{\mathrm{ZK}-3}$ & 1.5 & 92690 & 158 & 1.49 & 31 & 21 & 34 & 17 & 106 & 94 & 9 & 167 & 196 \\
\hline ZK-5 & 2.5 & 113623 & 241 & 1.55 & 30 & 19 & 27 & 21 & 155 & 95 & 11 & 137 & 200 \\
\hline ZK-8 & 4 & 103762 & 224 & 1.51 & 24 & 16 & 23 & 22 & 148 & 95 & 12 & 127 & 164 \\
\hline ZK-10 & 5 & 96706 & 223 & 1.43 & 36 & 25 & 37 & 23 & 156 & 92 & 18 & 138 & 168 \\
\hline ZK-12 & 6 & 80291 & 186 & 1.00 & 32 & 32 & 40 & 23 & 186 & 82 & 10 & 122 & 164 \\
\hline ZK-14 & 7 & 86305 & 182 & 3.17 & 26 & 8 & 30 & 21 & 57 & 128 & 11 & 236 & 185 \\
\hline ZK-17 & 11 & 90801 & 176 & 1.07 & 21 & 20 & 24 & 19 & 164 & 85 & 10 & 142 & 166 \\
\hline ZK-20 & 12.5 & 94372 & 214 & 0.87 & 19 & 22 & 20 & 23 & 246 & 84 & 11 & 113 & 131 \\
\hline ZK-22 & 13.5 & 91816 & 191 & 1.02 & 28 & 27 & 30 & 21 & 187 & 91 & 19 & 139 & 173 \\
\hline ZK-23 & 14 & 86026 & 182 & 1.10 & 37 & 34 & 43 & 21 & 165 & 90 & 10 & 115 & 168 \\
\hline ZK-26 & 15.25 & 79826 & 171 & 1.17 & 29 & 25 & 36 & 21 & 146 & 86 & 12 & 129 & 156 \\
\hline ZK-27 & 15.5 & 102655 & 209 & 0.86 & 43 & 50 & 42 & 20 & 243 & 77 & 9 & 88 & 157 \\
\hline ZK-28 & 15.75 & 109131 & 221 & 0.89 & 43 & 49 & 40 & 20 & 248 & 78 & 9 & 88 & 158 \\
\hline ZK-30 & 16.25 & 116885 & 260 & 1.37 & 23 & 17 & 20 & 22 & 190 & 74 & 12 & 87 & 184 \\
\hline ZK-32 & 16.75 & 120010 & 274 & 1.62 & 24 & 15 & 20 & 23 & 169 & 86 & 9 & 75 & 145 \\
\hline ZK-35 & 17.5 & 114482 & 281 & 0.66 & 38 & 58 & 34 & 25 & 426 & 85 & 10 & 110 & 202 \\
\hline ZK-37 & 18 & 75576 & 168 & 0.20 & 40 & 199 & 53 & 22 & 840 & 67 & 6 & 122 & 153 \\
\hline ZK-39 & 18.25 & 97538 & 219 & 0.32 & 29 & 90 & 29 & 22 & 684 & 81 & 6 & 140 & 207 \\
\hline ZK-41 & 18.75 & 96043 & 411 & 0.28 & 42 & 150 & 44 & 43 & 1468 & 81 & 11 & 114 & 156 \\
\hline ZK-42 & 19.25 & 95809 & 417 & 0.28 & 70 & 250 & 73 & 44 & 1489 & 78 & 10 & 107 & 171 \\
\hline ZK-43 & 19.5 & 107655 & 246 & 0.40 & 124 & 310 & 115 & 23 & 615 & 81 & 13 & 83 & 241 \\
\hline ZK-45 & 19.75 & 114811 & 271 & 0.21 & 324 & 1544 & 282 & 24 & 1290 & 79 & 16 & 76 & 129 \\
\hline ZK-47 & 20.25 & 106907 & 250 & 0.30 & 42 & 141 & 39 & 23 & 833 & 94 & 6 & 123 & 230 \\
\hline ZK-50 & 21 & 92401 & 209 & 0.45 & 39 & 87 & 42 & 23 & 464 & 93 & 7 & 161 & 162 \\
\hline ZK-52 & 21.5 & 97483 & 253 & 0.76 & 24 & 31 & 24 & 26 & 333 & 81 & 7 & 122 & 135 \\
\hline ZK-54 & 22 & 94306 & 241 & 0.72 & 26 & 37 & 28 & 26 & 335 & 79 & 7 & 105 & 148 \\
\hline ZK-56 & 22.5 & 89934 & 220 & 0.44 & 36 & 82 & 40 & 24 & 500 & 83 & 8 & 118 & 161 \\
\hline ZK-58 & 23 & 94185 & 237 & 0.41 & 14 & 35 & 15 & 25 & 578 & 82 & 10 & 111 & 168 \\
\hline ZK-61 & 23.75 & 72446 & 171 & 0.30 & 21 & 72 & 30 & 24 & 570 & 46 & 7 & 98 & 92 \\
\hline ZK-63 & 24.25 & 89518 & 203 & 0.26 & 42 & 161 & 47 & 23 & 781 & 98 & 8 & 164 & 180 \\
\hline ZK-65 & 24.75 & 90395 & 265 & 0.26 & 16 & 60 & 17 & 29 & 1019 & 86 & 10 & 148 & 157 \\
\hline ZK-67 & 25.25 & 85811 & 182 & 0.51 & 6 & 12 & 7 & 21 & 357 & 80 & 7 & 130 & 149 \\
\hline ZK-70 & 26 & 89655 & 207 & 0.26 & 8 & 29 & 8 & 23 & 796 & 82 & 9 & 155 & 166 \\
\hline ZK-72 & 26.5 & 82691 & 188 & 0.25 & 11 & 43 & 13 & 23 & 752 & 77 & 10 & 178 & 162 \\
\hline ZK-74 & 27 & 89460 & 213 & 0.52 & 11 & 21 & 12 & 24 & 410 & 75 & 8 & 118 & 159 \\
\hline ZK-77 & 27.75 & 92990 & 219 & 0.57 & 14 & 25 & 15 & 24 & 384 & 72 & 10 & 92 & 153 \\
\hline
\end{tabular}


Table DR2. Counts of total and tetrad spores from ZK4703.

\begin{tabular}{llllllll}
\hline Sample & Height $(\mathrm{m})$ & Total & Spore & Spore/total & Tetrads & Tetrads/total & Tetrad/spore \\
\hline ZK-5 & 2.50 & 199 & 190 & 0.9548 & 0 & 0.0000 & 0.0000 \\
ZK-7 & 3.50 & 70 & 67 & 0.9571 & 0 & 0.0000 & 0.0000 \\
ZK-10 & 5.00 & 74 & 72 & 0.9730 & 0 & 0.0000 & 0.0000 \\
ZK-12 & 6.00 & 81 & 79 & 0.9753 & 0 & 0.0000 & 0.0000 \\
ZK-14 & 7.00 & 90 & 90 & 1.0000 & 0 & 0.0000 & 0.0000 \\
ZK-17 & 11.00 & 168 & 168 & 1.0000 & 0 & 0.0000 & 0.0000 \\
ZK-26 & 15.25 & 74 & 66 & 0.8919 & 0 & 0.0000 & 0.0000 \\
ZK-28 & 15.75 & 204 & 194 & 0.9510 & 0 & 0.0000 & 0.0000 \\
ZK-30 & 16.25 & 195 & 193 & 0.9897 & 0 & 0.0000 & 0.0000 \\
ZK-34 & 17.25 & 196 & 164 & 0.8367 & 1 & 0.0051 & 0.0061 \\
ZK-37 & 18.00 & 298 & 168 & 0.5638 & 1 & 0.0034 & 0.0060 \\
ZK-40 & 18.50 & 339 & 301 & 0.9066 & 21 & 0.0633 & 0.0698 \\
ZK-41 & 18.75 & 340 & 326 & 0.9588 & 64 & 0.1882 & 0.1963 \\
ZK-42 & 19.25 & 204 & 200 & 0.9804 & 36 & 0.1765 & 0.1800 \\
ZK-43 & 19.50 & 152 & 121 & 0.7961 & 1 & 0.0066 & 0.0083 \\
ZK-45* & 19.75 & 61 & 51 & 0.8360 & 2 & 0.0327 & 0.0392 \\
ZK-53 & 21.75 & 87 & 49 & 0.3793 & 0 & 0.0000 & 0.0000 \\
ZK-65 & 24.75 & 147 & 34 & 0.2313 & 0 & 0.0000 & 0.0000 \\
ZK-77 & 27.75 & 171 & 48 & 0.2807 & 0 & 0.0000 & 0.0000 \\
ZK-79 & 28.25 & 142 & 45 & 0.3169 & 1 & 0.0070 & 0.0222 \\
\hline
\end{tabular}



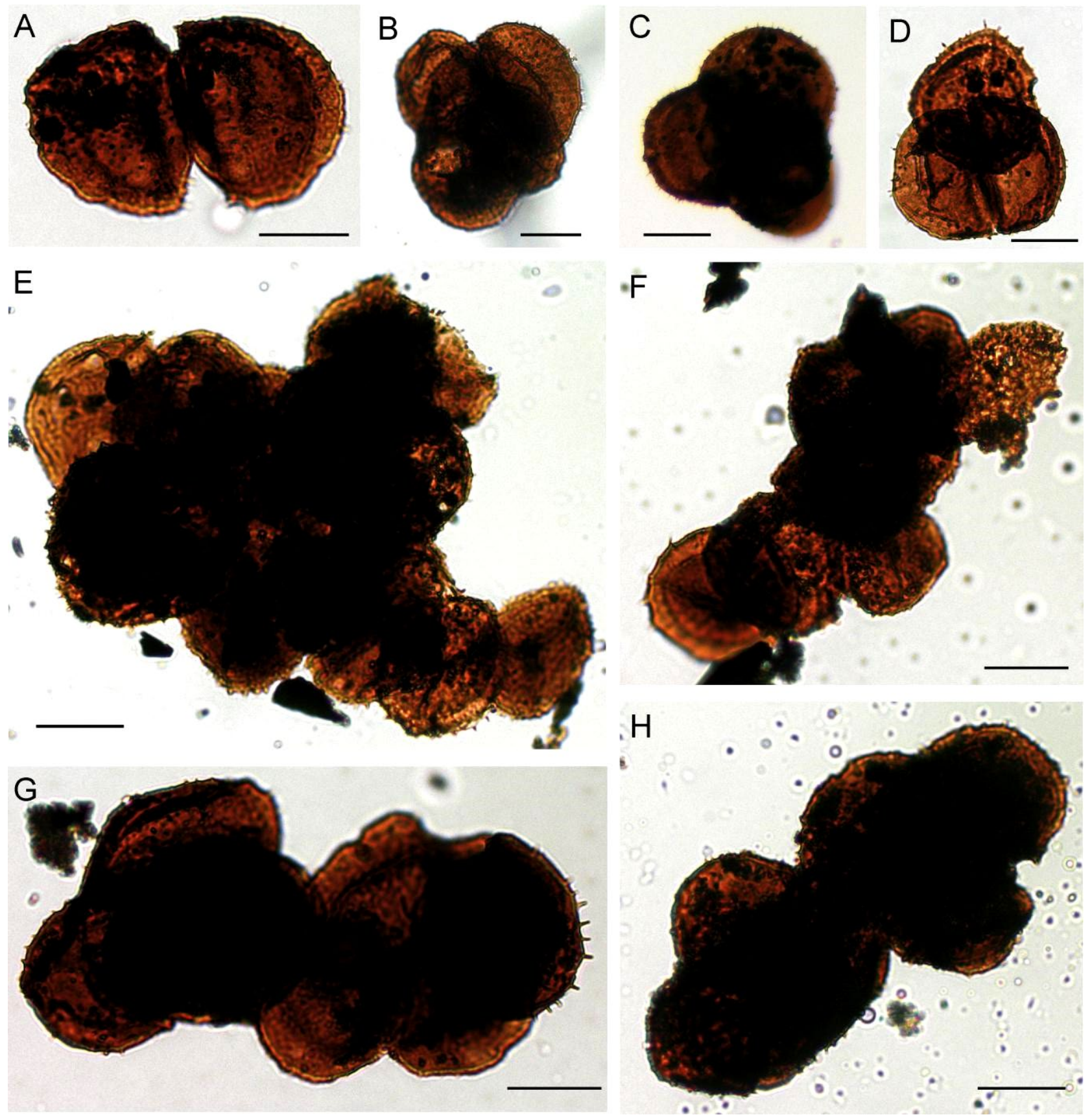

Figure DR1. A-D, Spore tetrads from ZK4703; E-H, masses of spores and tetrads stuck together. A-E are from sample ZK-41; F-H are from ZK-42. The scale bar is $20 \mu \mathrm{m}$. 